\title{
MODELO PARA O DESPACHO DE USINAS INDIVIDUALIZADAS NO PLANEJAMENTO HIDROTÉRMICO DE MÉDIO PRAZO BASEADO EM PNL
}

\author{
Tales P. Ramos* \\ tales.pulinhodengenharia.ufjf.br \\ Ivo C. Silva Junior* \\ ivo.junior@ufje.edu.br \\ Bruno H. Dias ${ }^{\dagger}$ \\ bdias@ieee.org \\ Rafael B. S. Brandi* \\ rafael.brandi@engenharia.ufjf.br
}

\author{
André L. M. Marcato* \\ andre.marcato@ufjf.edu.br \\ João A. Passos Filho* \\ joao.passos@ufjf.edu.br
}

Edimar J. de Oliveira*

edimar.oliveira@ufjf.edu.br

\author{
Anderson M. Iung $\ddagger$ \\ amiung@duke-energy.com
}

*Universidade Federal de Juiz de Fora - UFJF - MG

${ }^{\dagger}$ Universidade Federal Fluminense - UFF - RJ.

${ }_{\ddagger}^{\ddagger}$ Duke Energy - SP

\begin{abstract}
NLP Based Model for Individual Plant Dispatch in Long Term Hydrothermal Planning

This paper presents a method to the hydrothermal dispatch using optimization techniques based on non linear programming techniques. To do so, the expected cost-to-go functions from a long term operation plannning strategic decision model are used. This decision model is based on stochastic dual dynamic programming and energy equivalent reservoirs. The proposed method considers a set of historical water inflow scenarios to the hydroelectric reservoirs. Those scenarios are used to simulate the long term operation planning to a given horizon. The results obtained from this disaggregation model (MIUH) are compared with those from the model officially adopted in the Brazilian power system, SUISHI-O. The latter is based on operation heuristics aiming at operating the reservoir maintaining the water storage in similar levels, that is, trying to operate them in parallel.
\end{abstract}

Artigo submetido em 18/01/2011 (Id.: 1253)

Revisado em 06/04/2011, 08/08/2011, 09/12/2011

Aceito sob recomendação do Editor Associado Prof. Arturo Bretas
KEYWORDS: Power System Operation planning; hydrothermal dispatch, optimization, dynamic programming.

\section{RESUMO}

Este trabalho apresenta um modelo de despacho hidrotérmico à usinas individualizadas, utilizando métodos de otimização baseados em programação não linear. Para tanto, considera-se funções de custo futuro geradas por um modelo de decisão estratégica baseado em programação dinâmica e sistemas equivalentes de energia. O modelo proposto considera diversos cenários históricos de afluências hidrológicas às usinas hidrelétricas, os quais são simulados para um horizonte de planejamento da operação de médio/longo prazo. Os resultados obtidos através do modelo proposto, denominado Modelo Individualizado de Usinas Hidráulicas (MIUH), são comparados com os resultados obtidos a partir da utilização do modelo SUISHI-O adotado pelo Operador Nacional do Sistema Elétrico Brasileiro (ONS).

PALAVRAS-CHAVE: Planejamento da operação, despacho hidrotérmico, métodos de otimização, programação dinâmica. 


\section{INTRODUÇÃO}

O crescimento dos Sistemas Elétricos de Potência em tamanho e complexidade devido ao aumento da demanda de energia elétrica e à necessidade de maior confiabilidade dos mesmos, aliado à necessidade de redução de custos, resultou em uma interligação cada vez maior entre sistemas de geração já existentes. Os sistemas interligados são vantajosos na medida em que permitem ganhos energéticos através da coordenação da operação hidrotérmica, o que garante melhor aproveitamento hidrológico entre as bacias existentes. Com o aumento destas interligações, a operação coordenada do sistema torna-se bastante complexa, exigindo um planejamento detalhado de suas condições de operação para que o desempenho alcançado seja compatível com os requisitos de qualidade e confiabilidade.

Dentro deste contexto, o planejamento da operação de médio/longo prazo de sistemas hidrotérmicos tem por objetivo principal atender a demanda projetada de energia elétrica para um horizonte de até cinco anos (médio prazo) ou até 20 anos (longo prazo) com base mensal de discretização, considerando-se as incertezas da disponibilidade e os custos dos insumos utilizados (Fortunato, 1990). Desta forma, o problema consiste em determinar os montantes ótimos de geração hídrica e térmica, intercâmbios e déficits para todos os estágios do planejamento, visando minimizar o custo total esperado de operação, ao longo do horizonte de estudo e considerando-se a estocasticidade das afluências.

As três principais características deste problema são: $(i)$ a estocasticidade relacionada com a incerteza das afluências futuras aos reservatórios; (ii) o acoplamento espacial, onde a operação de uma usina hidrelétrica com reservatório a montante impacta a operação das usinas localizadas a jusante; (iii) o acoplamento temporal, que está relacionado com o impacto da decisão atual de geração no custo de operação dos estágios seguintes.

De uma forma geral, o processo de decisão, em cada estágio, consiste em escolher entre gerar energia de fontes hidráulicas, aumentando o custo de operação nos estágios seguintes (futuro), no caso de afluências desfavoráveis ou economizar água nos reservatórios, reduzindo o custo nos estágios posteriores e, consequentemente, aumentando o custo presente. Tradicionalmente, no Setor Elétrico Brasileiro (SEB), a modelagem deste processo é realizada através de Funções de Custo Futuro (FCFs) que são obtidas através de técnicas de otimização (Silva, 2001). Estas FCFs podem ser obtidas através da equação do corte de Benders realizado durante o processo de decomposição dinâmica dual estocástica (J.F. Benders, 1962).

Uma outra forma de obter as FCFs foi apresentada em (Dias et al., 2010) que utiliza a técnica de Programação Dinâmica
Estocástica (PDE) integrada com algoritmos de fechos convexos (Convex Hull).

Dentre as técnicas de otimização utilizadas no planejamento da operação de sistemas hidrotérmicos destacam-se as técnicas de programação dinâmica. A programação dinâmica é um processo sequencial de tomada de decisões que segue o "principio da otimalidade de Bellman" (Bellman, 1957). Tendo em vista as incertezas presentes no problema, tais como as afluências aos reservatórios, técnicas de programação estocástica (Birge e Louveaux, 1997) são utilizadas como em Santos et al (2009).

Por sua vez, a utilização da programação dinâmica resulta em um elevado esforço computacional devido ao número de estados operativos a serem analisados para a obtenção do custo ótimo de operação, resultando no chamado mal da dimensionalidade. Para contornar este problema são utilizadas modelagens baseadas na agregação das usinas hidrelétricas em sistemas equivalentes de energia, de modo a reduzir o espaço de busca e consequentemente o número de estados operativos a serem avaliados (Arvanitidis and Rosing, 1970).

Diversos trabalhos na literatura utilizam a PDE considerando-se a agregação de reservatórios para realização do planejamento de médio/longo prazo em reservatórios equivalentes e a seguir a desagregação dos montantes obtidos entre cada uma das usinas (Turgeon, 1998; Valdés, 1995). Um método de desagregação baseado em Redes Neurais para a desagregação é mostrado por Saad et al. (1994). Abordagens alternativas realizam o planejamento da operação a longo prazo de forma determinística, e então podem trabalhar com a representação individualizida das usinas, tal como visto em Azevedo et at. (2009) e mais recentemente em Zambelli (2010).

Uma técnica utilizada que suplanta a necessidade de discretização de todo o espaço de estados é a Programação Dinâmica Dual Estocástica (CEPEL, 2001; Pereira, 1989; Silva and Finardi, 2003). Particularmente, o modelo adotado pelo ONS para a obtenção das FCFs associadas a cada mês de estudo é denominado NEWAVE e utiliza a técnica de Programação Dinâmica Dual Estocástica (PDDE). Em todos estes modelos, o problema é dividido em duas etapas, as quais são descritas a seguir (Marcato, 2002):

1. Cálculo da Política Operativa: Um conjunto de cenários hidrológicos (históricos ou sintéticos) são utilizados para a construção das FCFs para cada estágio (ou período) de estudo.

2. Simulação da Operação: Um conjunto de cenários hidrológicos (históricos ou sintéticos) é utilizado para reproduzir a operação do sistema utilizando-se as FCFs produzidas na etapa de cálculo da política. Na simu- 
lação da operação, os problemas de otimização resolvidos têm base mensal e a energia armazenada no final do estágio é utilizada como energia armazenada inicial do estágio seguinte. O objetivo é produzir estatísticas para as diversas variáveis de decisão associadas ao problema (Geração Hidráulica, Geração Térmica, Intercâmbios Energéticos, Déficit de Energia, Energia Armazenada, Custo Marginal de Operação, etc.).

Portanto, em linhas gerais, como resultado da simulação da operação tem-se, para cada cenário hidrológico e mês de estudo, as metas de geração hidráulica e térmica de cada um dos subsistemas equivalentes de energia.

No entanto, no planejamento da operação de médio/longo prazo também é necessária a determinação dos montantes de geração individuais de cada usina hidrelétrica. Este procedimento deve ser realizado considerando-se as restrições associadas a operação individualizada das usinas hidrelétricas dos subsistemas equivalentes. Para tanto, o ONS utiliza o modelo SUISHI-O, desenvolvido pelo CEPEL (CEPEL, 2007) capaz de considerar as FCFs geradas pelo modelo NEWAVE e através de procedimentos heurísticos, baseados na busca pela operação em paralelo dos reservatórios, desagrega a geração hidráulica dos subsistemas equivalentes entre suas usinas hidrelétricas. Além disso, verifica se é possível a obtenção de uma solução factível. (CEPEL, 2007).

O presente trabalho propõe a utilização de uma nova metolodologia para a determinação da estratégia de operação das usinas hidroelétricas, denominada de Modelo Individualizado de Usinas Hidráulicas (MIUH). Tal metodologia é baseada em métodos de otimização que utilizam programação não linear ao invés dos procedimentos heurísticos, que são utilizados pelo modelo SUISHI-O. Será apresentado um estudo de caso com o Sistema Interligado Nacional (SIN), onde são comparados os resultados obtidos pelos modelos SUISHI-O e MIUH. Estes resultados são realizados utilizando-se as FCFs calculadas pelo modelo NEWAVE.

\section{METODOLOGIA ATUAL}

Como descrito na Seção anterior, o processo de simulação da operação realizado pelo modelo NEWAVE é baseado em sistemas equivalentes de energia. Este modelo leva em consideração o crescimento do mercado de energia, a entrada e saída de operação de empreendimentos de geração e a expansão das interconexões entre os subsistemas entre outros aspectos.

Atualmente, o modelo NEWAVE considera a agregação das usinas hidrelétricas em reservatórios equivalentes de energia representados por quatro grandes subsistemas, são eles: Norte, Nordeste, Sul, Sudeste/Centro-Oeste, conforme mos- trado na Figura 1. Para cada cenário hidrológico selecionado (histórico ou sintético) é realizada a operação dos sistemas equivalentes de energia para todo o horizonte de estudo.

Entretanto, não é possível garantir que a meta de geração estabelecida para os subsistemas equivalentes de energia seja factível quando se considera as usinas de forma individualizadas, podendo ocorrer uma das três situações a seguir: $(i) \mathrm{A}$ meta de geração hidráulica estabelecida para o sistema equivalente é muita alta para ser atingida pelas usinas quando operadas individualmente; (ii) A meta de geração hidráulica é baixa frente ao estoque disponível de água e afluência nos reservatórios ocasionando um excesso de água que não poderia ser estocado; (iii) A meta de geração hidráulica para o subsistema equivalente pode ser perfeitamente atendida pelo conjunto de suas usinas hidrelétricas.

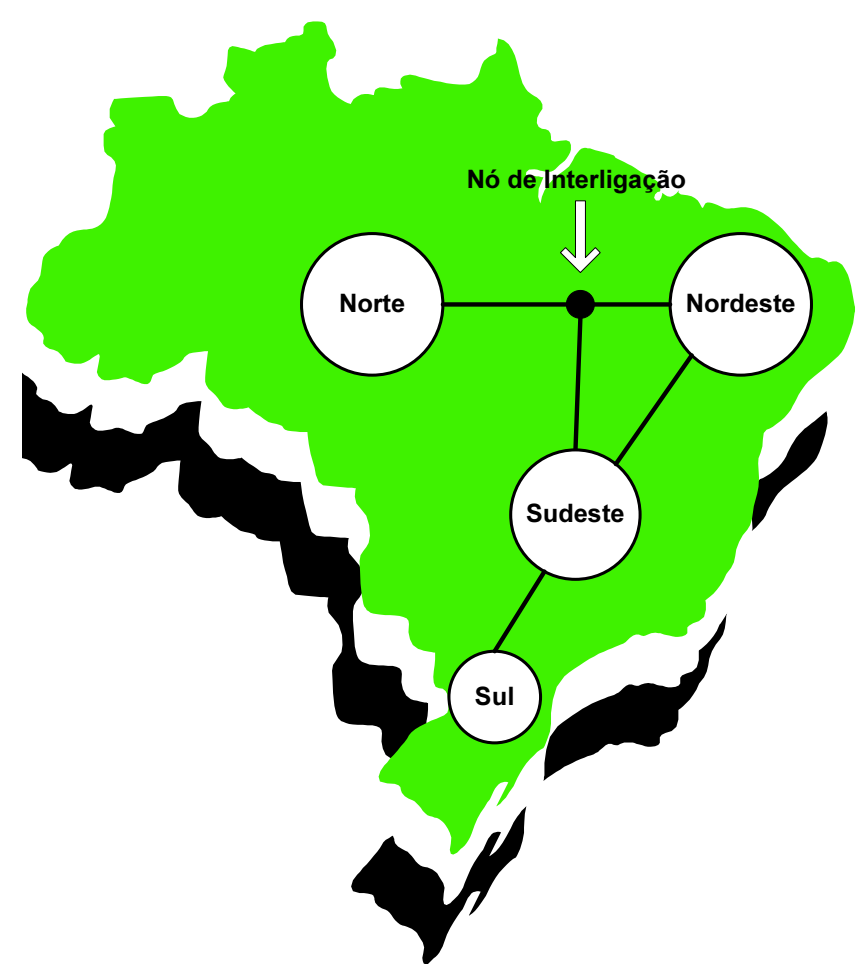

Figura 1: Diagrama dos subsistemas do SEB

É importante destacar que quando ocorrem as situações ( $i$ ) ou (ii) o despacho dos sistemas equivalentes é refeito, considerando-se restrições adicionais relacionadas a máxima geração hidráulica ou armazenamento máximo dos subsistemas equivalentes. Este processo é repetido sucessivas vezes 
até que as metas de todos os subsistemas equivalentes possam ser atendidas pelas suas usinas individualizadas, situação (iii). Este processo é ilustrado no Fluxograma do programa SUISHI-O, mostrado na Figura 2. Observa-se que este modelo é composto por dois módulos, que serão sucintamente descritos a seguir.

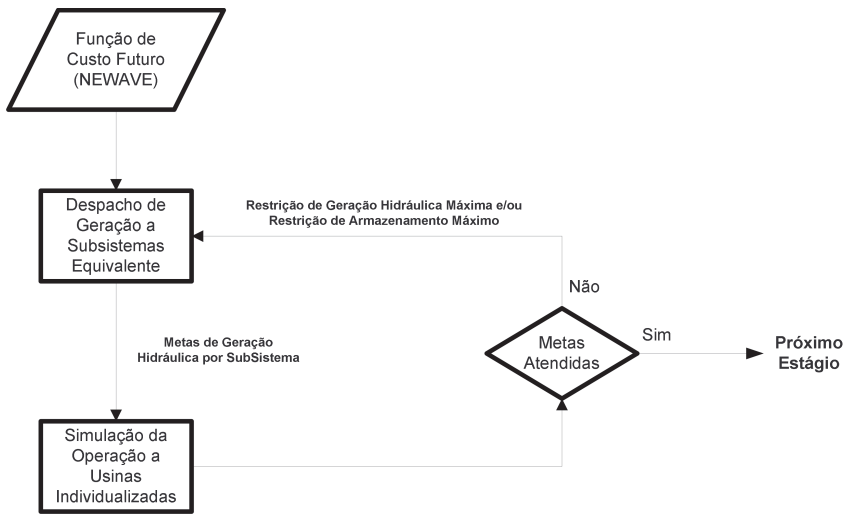

Figura 2: Fluxograma do programa SUISHI-O.

O primeiro módulo realiza, a partir das FCFs geradas no modelo de médio prazo, o balanço hidrotérmico entre os subsistemas equivalentes de energia através da resolução de um problema de programação linear. Neste módulo são definidas as metas de geração de cada subsistema.

As metas definidas são fornecidas ao segundo módulo, simulação da operação à usinas individualizadas, que através de regras heurísticas realiza os despachos de cada usina do sistema, visando o cumprimento das metas estabelecidas no módulo anterior. Estas heurísticas tentam manter o volume dos reservatórios dentro de uma mesma faixa de operação, ou seja, busca operar as usinas em paralelo (CEPEL, 2007).

As heurísticas operativas representam as não linearidades inerentes ao despacho hidráulico à usinas individualizadas, impostas pela variação da produtibilidade em função dos polinômios cota-volume, cota-área e vazão-nível jusante (Marcato, 2002). Adicionalmente, tais heurísticas permitem incorporar as restrições impostas pela operação em cascatas dos aproveitamentos hidráulicos, volume mínimo para vertimento, usos múltiplos da água, vazão mínima obrigatória, volume evaporado entre outras.

\section{MODELO PROPOSTO}

O modelo proposto neste trabalho consiste na utilização de métodos de otimização baseados em programação não-

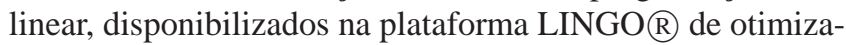
ção (LINGO, 2008). Este solver utiliza a técnica de programação linear sucessiva (SLP) e a técnica do gradiente reduzido generalizado (GRG).
Os resultados gerados com o solver são, principalmente, os valores de geração hidráulica e térmica de cada usina existente no sistema, os valores dos intercâmbios entre os subsistemas, o déficit de cada subsistema para cada período de estudo e cenário hidrológico.

O objetivo principal deste trabalho consiste em minimizar o custo de operação no horizonte desejado, levando-se em consideração todas as restrições físicas e operativas do sistema através de um Modelo Individualizado de Usinas Hidráulicas (MIUH). A partir das FCFs calculadas por um modelo de decisão estratégica tal como o NEWAVE ou CHULL (Dias et al., 2010), o problema pode ser desacoplado para obter a solução em estágios mensais.

Neste sentido, a função objetivo do problema consiste em minimizar a soma dos custos imediatos de operação de cada mês (geração térmica e custo de déficits de energia) e o custo futuro. O custo futuro representa o valor esperado de operação considerando-se a decisão operativa (geração térmica e déficit) do estágio corrente. Desta forma, a função objetivo a ser minimizada em cada mês $(t)$ de estudo é dada pela equação (1).

$$
\begin{aligned}
& \operatorname{Min} \sum_{s=1}^{N S I S}\left(\sum_{n=1}^{N G T_{s}} C T_{n, s}^{t} \cdot G T_{n, s}^{t}\right)+ \\
& +\left(C D_{s} \cdot D E F_{s}^{t}\right)+\frac{1}{1+\beta} \alpha^{t+1}
\end{aligned}
$$

onde:

$C T_{n, s}^{t}$ : Custo geração térmica associado à unidade termoelétrica $n$ do subsistema $s$, no estágio $t$ (\$/MWmês);

$G T_{n, s}^{t}$ : Geração da unidade termelétrica $n$ do subsistema $s$, no estágio $t$ (MWmês);

$C D_{s}$ : Custo de déficit associado ao subsistema $s$ (\$/MWmês);

$D E F_{s}^{t}$ : Déficit no estágio $t$, associado ao subsistema $s$ (MWmês);

NSIS: Número de subsistemas;

$N G T_{s}$ : Número de unidades termoelétricas do subsistema $s$;

$\alpha^{t+1}$ : Função de custo futuro pré-calculada (NEWAVE ou CHULL ou outro modelo de decisão), (\$);

$\beta$ : Taxa de desconto. 
A seguir é descrita a restrição responsável pelo acoplamento do despacho a usinas individualizadas com a FCF baseada em subsistemas equivalentes (modelo de decisão estratégico). Além disso, são apresentadas as restrições físicas e operacionais do problema individualizado.

\section{Função de Custo Futuro}

As restrições relativas ao custo futuro representam o acoplamento temporal das decisões presentes, ou seja, o uso da geração hidráulica no mês presente representa um aumento no custo de operação nos meses subsequentes devido ao aumento da probabilidade de necessidade de geração térmica no futuro, ocasionado pela redução do volume de água disponível nos reservatórios no estágio atual. Estas funções relacionam este impacto no custo da operação do sistema (Silva, 2001).

A equação (2) tem grande importância no problema, pois ela acopla o despacho à usinas individualizadas com o despacho baseado em subsistemas equivalentes de energia. Esta equação representa os cortes de Benders (J.F. Benders, 1962) determinados durante o processo de programação dinâmica dual estocástica. Uma aplicação desta técnica pode ser encontrada em (Pereira, M. V. F., 1989). Desta forma, evita-se a necessidade de utilizar-se um processo iterativo análogo ao utilizado pelo modelo SUISHI-O, ou seja, para cada estágio, é resolvido apenas um problema de programação não linear que realiza o despacho à usinas individualizadas acessando uma FCF baseada em subsistemas equivalentes de energia. Para cada corte da FCF deve ser incluída uma restrição dada pela equação (2). A produtibilidade acumulada utilizada na equação (3) é calculada a partir da soma da produtibilidade da usina e todas as suas jusantes em cascata até o oceano (Marcato, 2002).

$$
\begin{aligned}
& \alpha^{t+1} \geq w_{j}^{t}+\sum_{s=1}^{N S I S}\left[\left(\pi_{E A}\right)_{j, s}^{t+1} \cdot E A R M_{s}^{t+1}\right]+\ldots \\
& +\sum_{s=1}^{N S I S} \sum_{p=1}^{N A R P_{s}}\left[\left(\pi_{E A F_{p}}\right)_{j, s}^{t+1} \cdot E N A_{s}^{t-p+1}\right] \mathrm{j}=1, \ldots \mathrm{ncb}
\end{aligned}
$$

$$
\begin{aligned}
E A R M_{s}^{t} & =\sum_{h=1}^{N D A M_{s}} \frac{V A_{h, s}^{t} \cdot\left(\rho^{\text {acum }}\right)_{h, s}^{t}}{\text { fator }} \\
E N A_{s}^{t} & =\sum_{h=1}^{N G H_{s}} V A Z N_{h, s}^{t} \cdot \rho_{h, s}^{t}
\end{aligned}
$$

$w_{j}^{t}$ : Termo constante $j$-ésimo corte de Benders, no estágio $t,(\$)$;

$\left(\pi_{E A}\right)_{j, s}^{t+1}$ : Coeficiente do $j$-ésimo corte construído no estágio $t+1$ associado ao armazenamento do subsistema $s$

$E A R M_{s}^{t+1}$ : Energia Armazenada no subsistema $s$, no estágiot +1 (MWmês);

$N A R P_{s}$ : Ordem máxima do modelo PAR(p) para o subsistema $s$;

$\left(\pi_{E A F_{p}}\right)_{j, s}^{t+1}:$ Coeficiente do $j$-ésimo corte construído no estágio $t+1$, associado à afluência do $p$-ésimo estágio passado e ao subsistema $s$;

$E N A_{s}^{t-p+1}$ : Energia Natural Afluente ao subsistema $s$ associada ao estágio $t-p+1$ (MWmês);

$n c b$ : Número de cortes de Benders;

$N D A M_{s}$ : Número de usinas com reservatórios do subsistema $s$;

$V A_{h, s}^{t}$ : Volume armazenado da usina hidráulica $h$, pertencente ao subsistema $s$, no estágio $t\left(\mathrm{hm}^{3}\right)$;

$\left(\rho^{\text {acum }}\right)_{h, s}^{t}: \quad$ Produtibilidade acumulada associada à usina $h$, pertencente ao subsistema $s$, no estágio $t$ (MWmês $/ \mathrm{m}^{3} / \mathrm{s}$ ).

fator: Fator de conversão de $\mathrm{m}^{3} / \mathrm{s}$ para $\mathrm{hm}^{3}$.

$V A Z N_{h, s}^{t}$ : Vazão natural à usina hidráulica $h$, pertencente ao subsistema $s$, no estágio $t\left(\mathrm{~m}^{3} / \mathrm{s}\right)$;

$\rho_{h, s}^{t}:$ Produtibilidade associada à usina $h$, pertencente ao subsistema $s$, no estágio $t$ (MWmês $/ \mathrm{m}^{3} / \mathrm{s}$ ).

$N G H_{s}$ : Número de usinas hidráulicas do subsistema $s$;

\section{Equação de Atendimento a Demanda:}

Esta restrição representa $o$ atendimento ao mercado de energia através das gerações das usinas hidráulicas e térmicas. Adicionalmente, devem ser contabilizados os valores de intercâmbio entre subsistemas (troca de energia), além de um possível déficit no sistema. A equação (5) mostra a restrição de atendimento a demanda inserida no modelo MIUH.

$$
\begin{aligned}
& \sum_{n=1}^{N G T_{s}} G T_{n, s}^{t}+\sum_{h=1}^{N G H_{s}} \rho_{h . s} \cdot Q T U R_{h, s}^{t}+\sum_{s s=1}^{N S I S} I N T_{s s, s ; s \neq s s}^{t}-\ldots \\
& -\sum_{s s=1}^{N S I S} I N T_{s, s s ; s \neq s s}^{t}+D E F_{s}^{t}=D E M A N D A_{s}^{t}
\end{aligned}
$$

onde: 
onde:

$Q T U R_{h, s}^{t}$ : Vazão turbinada na unidade hidráulica $h$, no estágio $t$ pertencente ao subsistema $s\left(\mathrm{~m}^{3} / \mathrm{s}\right)$;

$I N T_{s s, s}^{t}$ : Intercâmbio de energia que chega ao subsistema $s$ a partir do sistema $s s$, no estágio $t$ (MWmês);

$I N T_{s, s s}^{t}$ : Intercâmbio de energia que chega ao subsistema ss a partir do sistema $s$, no estágio $t$ (MW.mês);

$D E M A N D A_{s}^{t}$ : Demanda no subsistema $s$ a ser atendida no estágio $t$ (MWmês).

\section{Equação de Balanço Hídrico:}

O volume do reservatório no final do período de estudo deve ser igual ao volume no início do período adicionado à vazão natural incremental ao reservatório mais o volume deplecionado nos reservatórios à montante, subtraído do volume turbinado, vertido e evaporado neste mesmo reservatório. Esta restrição é mostrada pela equação (6).

$$
\begin{aligned}
& V A_{h, s}^{t+1}=V A_{h, s}^{t}+\text { fator } \cdot V A Z I N C_{h, s}^{t}-V E V A P_{h, s}^{t}+\ldots \\
& -V T_{h, s}^{t}-V V_{h, s}^{t}+\sum_{r \in M_{h}}\left(V T_{r, s}^{t}+V V_{r, s}^{t}\right)
\end{aligned}
$$

onde: incremental corresponde à vazão natural do reservatório subtraída das vazões naturais das usinas imediatamente a montante. Para uma usina de cabeceira, a vazão natural é igual à vazão incremental.

\section{Restrições de canalização:}

Estas restrições, mostradas através das equações (7) a (11), representam os limites operativos dos geradores, bem como os limites possíveis de intercâmbio entre os subsistemas.

$$
\begin{gathered}
\underline{V T}_{h, s}^{t} \leq V T_{h, s}^{t} \leq \overline{V T}_{h, s}^{t} \\
\underline{V A_{h, s}^{t}} \leq V A_{h, s}^{t} \leq \overline{V A}_{h, s}^{t} \\
\underline{V V}{ }_{h, s}^{t} \leq V V_{h, s}^{t} \leq \overline{V V}_{h, s}^{t} \\
\underline{G T}_{n, s}^{t} \leq G T_{n, s}^{t} \leq \overline{G T}_{n, s}^{t} \\
\underline{I N T_{s, s s}^{t}} \leq I N T_{s, s s}^{t} \leq \overline{I N T}_{s, s s}^{t}
\end{gathered}
$$

onde:

$V A_{h, s}^{t}$ : Volume armazenado da usina hidráulica $h$ do subsistema $s$, no início do estágio $t\left(\mathrm{hm}^{3}\right)$;

$V A Z I N C_{h, s}^{t}$ : Vazão incremental afluente da hidrelétrica $h$ do subsistema $s$, no estágio $t\left(\mathrm{~m}^{3} / \mathrm{s}\right)$;

$V E V A P_{h, s}^{t}$ : Volume evaporado da hidrelétrica $h$ pertencente ao subsistema $s$, no estágio $t\left(\mathrm{hm}^{3}\right)$;

$V T_{h, s}^{t}$ : Volume turbinado da hidrelétrica $h$ pertencente ao subsistema $s$, no estágio $t\left(\mathrm{hm}^{3}\right)$;

$V V_{h, s}^{t}:$ Volume vertido da hidrelétrica $h$ pertencente ao subsistema $s$, no estágio $t\left(\mathrm{hm}^{3}\right)$;

$M_{h}$ : Conjunto das hidrelétricas imediatamente a montante da usina $h$.

Define-se vazão natural como sendo a vazão média mensal, em $\mathrm{m}^{3} / \mathrm{s}$, considerando-se a não existência de nenhum reservatório construído ao longo da cascata. No SEB existe um histórico de vazões naturais desde janeiro do ano de 1931 para todos os aproveitamentos hidráulicos. A vazão natural
$\overline{V T}_{h, s}^{t}, \underline{V T}_{h, s}^{t}$ : Limites máximo e mínimo do volume turbinado da usina hídrica $h$, do subsistema $s$, no estágio t. O limite máximo de turbinamento é recalculado para período de estudo em função do volume armazenado no final do período anterior $\left(\mathrm{hm}^{3}\right)$;

$\overline{V A}_{h, s}^{t}, \underline{V A}_{h, s}^{t}$ : Limites máximo e mínimo de armazenamento da usina hídrica $h$, do subsistema $s$, no estágio $t\left(\mathrm{hm}^{3}\right)$;

$\overline{V V}_{h, s}^{t}, \underline{V V}_{h, s}^{t}$ : Limites máximo e mínimo de vertimento da usina hídrica $h$, do subsistema $s$, no estágio $t\left(\mathrm{hm}^{3}\right)$;

$\overline{G T}_{n, s}^{t}, \underline{G T}_{n, s}^{t}$ : Limites máximo e mínimo de geração da usina térmica $n$, subsistema $s$, no estágio $t$, (MWmês);

$\overline{I N T}_{s, s s}^{t}, \underline{I N T}_{s, s s}^{t}$ Limites máximo e mínimo de intercâmbio do subsistema $s$ para o ss, no período $t$ (MWmês).

\section{Produtibilidade das UHEs:}

A produtibilidade $(\rho)$, no modelo proposto, é representada através de uma restrição não linear, equação (12), sendo função dos seguintes parâmetros: (i) Polinômio Cota Montante 
- Volume Armazenado (PCM), equação (14); (ii) Polinômio Cota Jusante - Vazão Defluente $(P C J)$, equação (15); (iii) Produtibilidade específica $\left(\rho^{e s p}\right)$; (iv) Perdas Hidráulicas $(P H)$. A equação (13) é utilizada para calcular o variação média do volume armazenado durante o mês, o qual é utilizado no PCM. Os parâmetros dos polinômios PCM e PCJ, a produtibilidade específica e a perda hidráulica são disponibilizados para cada usina hidrelétrica pelo Operador Nacional do Sistema (ONS, 2011).

$$
\begin{gathered}
\rho=\rho^{e s p} \cdot(P C M-P C J-P H) \\
V_{m}=\frac{V A_{t+1}+V A_{t}}{2} \\
P C M=a+b \cdot V_{m}+c \cdot V_{m}{ }^{2}+d \cdot V_{m}{ }^{3}+e \cdot V_{m}{ }^{4} \\
P C J=x+y \cdot Q D E F+w \cdot Q D E F^{2}+q \cdot Q D E F^{3}+\ldots \\
+z \cdot Q D E F^{4}
\end{gathered}
$$

Onde:

$Q D E F$ : Vazão defluente da usina $\left(\mathrm{m}^{3} / \mathrm{s}\right)$.

Neste trabalho propõe-se a representação do polinômio cotajusante através de uma função logística sigmoide. Esta representação é necessária para o melhor desempenho do modelo de otimização não linear. Este assunto será explorado através do estudo das principais características da função logística sigmoide mostrado a seguir.

\section{Função Logística Sigmoide:}

Um dos fatores característicos das unidades hidráulicas é a relação entre a cota de nível jusante do reservatório e a vazão defluente, sendo esta composta pela soma da vazão turbinada e vertida. Em linhas gerais, a cota de nível jusante do reservatório é função da vazão defluente e influencia na altura de queda e na produtibilidade, como mostrado nas equação (12) a (15).

Destaca-se que os coeficientes do polinômio de quarto grau $P C J$ representam características individuais de cada reservatório. Sendo estes obtidos a partir de medições realizadas à jusante do reservatório para diversas condições de operação, ou seja, diferentes observações de vazão defluente e sua respectiva cota de jusante (Hidalgo, 2009).
Como as defluências mínima e máxima utilizadas para obtenção destes pontos de ajuste nem sempre correspondem ao intervalo real de operação, o polinômio ajustado pode ter comportamento indesejado fora destes limites. Este comportamento interfere diretamente no processo de otimização, e, em algumas situações, faz com que o processo de convergência fique comprometido.

Um exemplo destes polinômios pode ser visto na Figura 3, referente à UHE LIMOEIRO (usina "Armando de Salles Oliveira"). O polinômio vazão nível jusante deve se comportar como uma função crescente entre a defluência mínima e máxima da Usina Hidrelétrica. Nesta usina, observa-se que a partir da defluência $900 \mathrm{~m}^{3} / \mathrm{s}$, a função passa a ser decrescente. Este comportamento produz um sinal contrário à realidade, onde aumentando-se a defluência ocorreria um aumento na altura de queda. Para contornar esse tipo de problema o modelo SUISHI-O adota uma heurística em que polinômios que decrescem a partir de um dado ponto máximo a cota é mantida constante a partir desse ponto, assegurando que a função de canal de fuga nunca será decrescente.

Com isto, propõe-se a utilização de uma nova forma de modelagem destes polinômios, utilizando funções logísticas sigmoides. Estas funções podem ser descritas através da equação 16 , onde é representada a função logística sigmoide (Haykin, 2004).

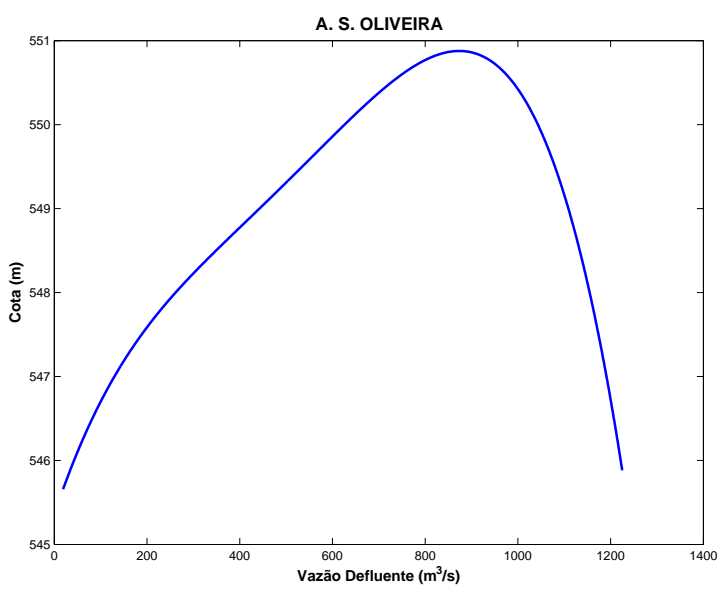

Figura 3: Exemplo de Polinômio Cota Jusante - Vazão Defluente.

$$
Y(t)=A+\frac{C-A}{1+e^{-B(t-M)}}
$$

Para cada polinômio foram adotados vinte pontos igualmente amostrados variando a partir da vazão defluente mínima até a defluência máxima (Fortunato, 1990) da usina em estudo. A partir destes pontos ajusta-se a função logistica sigmoide 
para cada usina hidrelétrica do sistema. Os parâmetros $B$ e $M$ foram calculados através de algoritmos genéticos visando a minimização do erro entre as curvas (polinômio de quarto grau e logística sigmoide), e os parâmetros $A$ e $C$ são constantes, cujos valores são iguais, respectivamente, aos valores mínimos e máximos do polinômio original, equação .

Na Figura 4 é mostrado o ajuste para a UHE A.S.OLIVEIRA e na Figura 5 o ajuste para a usina Furnas obtidos para os polinômios.

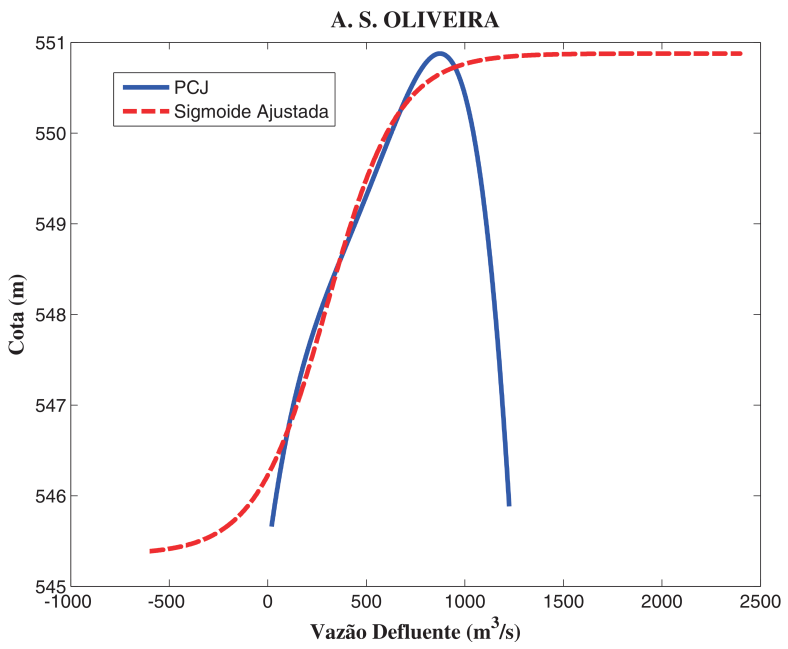

Figura 4: Sigmoide ajustada para a UHE A.S.OLIVEIRA

Como pode ser observado na procurou-se reproduzir o $P C J$ no intervalo de interesse para o problema. A vantagem na utilização deste tipo de função é sua "saturação" natural nos limites da região de interesse, evitando-se desta forma que os métodos de otimização busquem solução em pontos fora do intervalo de operação real da usina.

O tratamento do $P C J$ via sigmoide é adequado para utilização de solvers comerciais de otimização devido ao fato de não ser possível fixar uma curva no ponto de saturação. É importante destacar que os parâmetros da sigmoide são calculados uma única vez para cada usina e armazenado em um arquivo de dados. Desta forma, o tempo computacional para o ajuste das sigmoides não impacta na modelagem proposta.

Os parâmetros de ajuste das sigmoides obtidos para todas as usinas do SEB são apresentados no Apêndice A, Tabela A1. O erro médio percentual máximo encontrado no ajuste da sigmoide em relação ao ajuste polinomial foi de 1,48\% para a Usina de Tucuruí, calculado utilizando 1000 pontos de ajuste. $\mathrm{O}$ erro máximo encontrado dentre todos os pontos deste ajuste foi de $12,5 \%$ para o ponto de defluência mínima, correspondente a uma cota de 4 metros. No entanto, erros com esta ordem de grandeza ocorrem com uma frequência

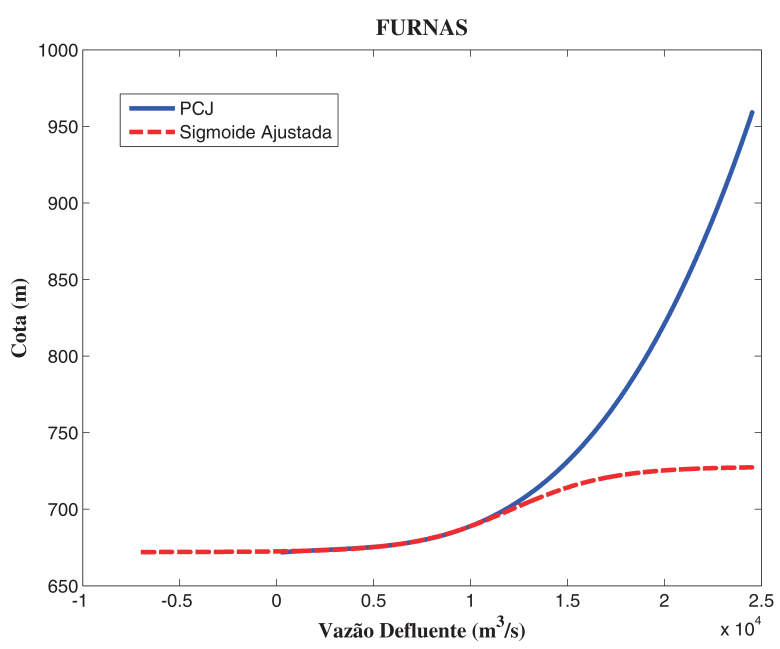

Figura 5: Sigmoide ajustada para a UHE FURNAS

muito pequena, visto que só são observados quando a defluência da usina é próxima ao valor mínimo ou máximo.

\section{Volume Mínimo para Vertimento:}

Esta restrição representa a cota do vertedouro dos reservatórios conforme especificado no conjunto de dados das usinas. A sua função é evitar que, no modelo de otimização proposto - MIUH, ocorra vertimento com o nível do reservatório abaixo do volume mínimo para vertimento associado à cota do vertedouro. Esta representação é importante uma vez que, em determinadas condições operativas, pode ser necessário realizar vertimento sem que o reservatório esteja com seu volume armazenado máximo. Neste sentido, define-se como volume mínimo para vertimento todo o volume do reservatório que está abaixo da cota do vertedouro da usina. A inequação proposta neste trabalho é apresentada em (17).

$$
\begin{aligned}
& V V_{h, s}^{t} \leq V A_{h, s}^{t}+\text { fator } \cdot V A Z I N C_{h, s}^{t}+\ldots \\
& +\sum_{r \in M_{h}}\left(V T_{r, s}^{t}+V V_{r, s}^{t}\right)-V T_{h, s}^{t}-V V M I N_{h, s}^{t}
\end{aligned}
$$

onde:

$V V M I N_{h, s}^{t}$ Volume mínimo para vertimento da usina hidráulica $\mathrm{h}$, no estágio $\mathrm{t}$, pertencente ao subsistema $s\left(h m^{3}\right)$;

\section{RESULTADOS}

Esta seção tem por objetivo principal descrever os resultados obtidos com a utilização do modelo proposto em um caso 
real do SEB. Os resultados que serão descritos a seguir são relativos à simulação da operação do sistema em um período de cinco anos de estudo. Os resultados apresentados são referentes a média de 60 cenários hidrológicos obtidos do histórico de vazões, sendo que o primeiro cenário de afluência corresponde ao ano de janeiro de 1931 a dezembro de 1935 do histórico de vazões, o segundo, de janeiro de 1932 a dezembro de 1936, e assim por diante.

Os dados do SEB que foram utilizados nesta simulação são referentes ao PMO (Programa Mensal de Operação) de janeiro de 2011 (ONS, 2011), que podem ser obtidos a partir do endereço eletrônico da Câmara de Comercialização de Energia Elétrica (www.ccee.org.br). Estes dados foram modificados de forma a considerar apenas um único patamar de carga, com taxa de desconto nula e desconsiderando-se os devios de água.

A Figura 6 mostra a comparação da evolução da Energia Armazenada (EARM) do sistema ao longo do período de estudo. É possível verificar que o MIUH conseguiu ao longo de todo o período a manutenção de níveis superiores de EARM em relação ao programa SUISHI-O.

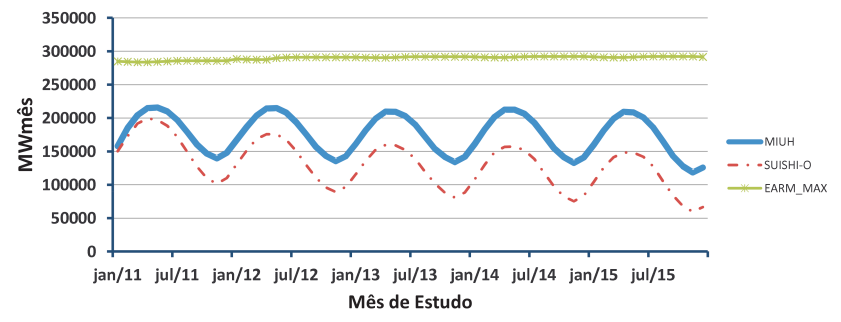

Figura 6: Evolução da Energia Armazenada do Sistema

As Figuras 7 a 10 mostram a comparação entre os dois modelos para cada um dos subsistemas (Sudeste, Sul, Nordeste e Norte). É possível verificar que nas Figuras 8, 9 e 10, a EARM do modelo proposto sempre se mantém em níveis superiores ao modelo SUISHI-O, enquanto que para o subsistema Norte, Figura 11, ocorreu uma alternância de comportamento. Isto porque no modelo MIUH o otimizador nãolinear conseguiu manter as usinas operando, em média, com uma produtibilidade superior em relação ao obtidos pelas heurísticas do modelo SUISHI-O.

As Figuras de 11 a 14 mostram a comparação da geração térmica e hidráulica para cada subsistema. Pode-se observar que o MIUH apresenta, em geral, menor utilização de geração térmica resultando em maior benefício para o SIN. No entanto, verifica-se que a ordem de grandeza e o comportamento sazonal da geração térmica e hidráulica são coerentes.

A menor utilização de geração térmica no despacho calculado pelo modelo MIUH resulta em um Custo Marginal de

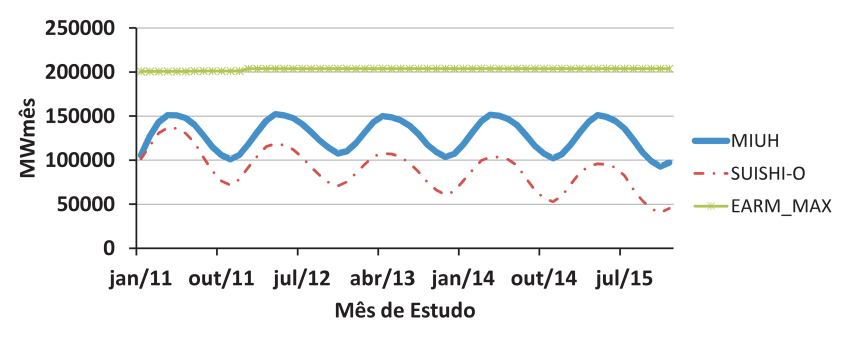

Figura 7: Energia Armazenada - SE

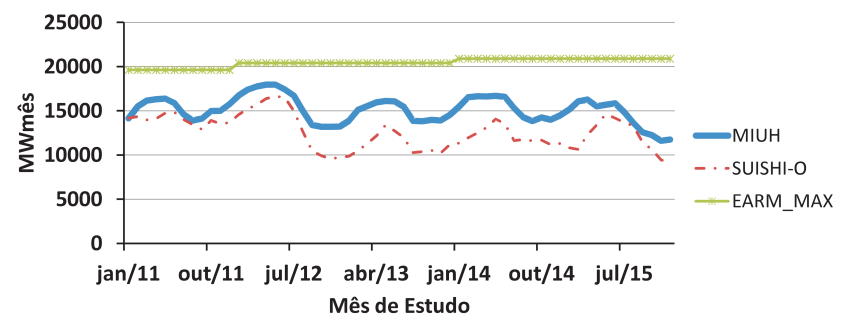

Figura 8: Energia Armazenada - S

Operação (CMO) médio menor e com menos variabilidade. A Figura 15 mostra a evolução do CMO médio do subsistema Sudeste. Os resultados obtidos para os demais subsistemas apresentam comportamento semelhante.

Pode ser observado a partir da Figura 15 que no primeiro mês de operação a energia armazenada média final é um pouco maior no modelo MIUH resultando em um CMO médio maior. Neste mês inicial ambos os modelos possuem produtibilidades iguais, mesmo volume inicial. Outro aspecto observado nesta figura, em relação aos meses subsequentes, é que o modelo SUISHI-O termina cada mês com armazenamento final menor e com o CMO médio maior. Isto ocorre devido a proposta de operação em paralelo dos reservatórios, a qual não existe no modelo MIUH. Ou seja, o modelo proposto MIUH é menos restritivo que o SUISHI-O.

Para verificar o impacto da utilização da função sigmoide na representação da variação da cota de jusante, a energia armazenada final foi re-calculada utilizando-se os polinômios de cota jusante - vazão defluente e cota montante - volume armazenado e os volumes armazenados finais, turbinamentos e vertimentos obtidos pelo MIUH. A Figura 16 mostra que a utilização da função sigmóide é adequada para este problema de otimização à usinas individualizadas tendo em vista a aderência entre os resultados.

Para mostrar os resultados da política de operação de forma individualizada foram escolhidas três usinas hidrelétricas do rio Paranaíba mostradas na Figura 17. 


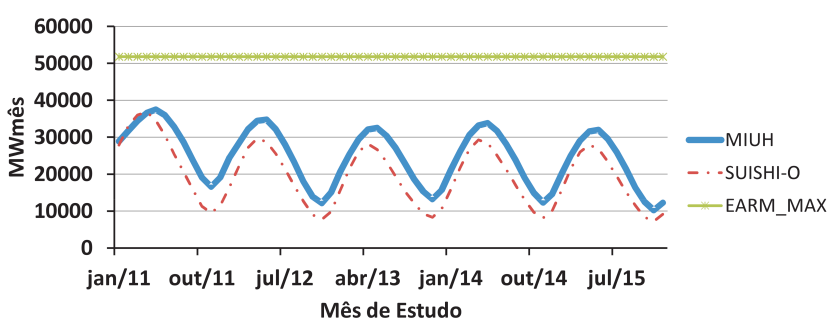

Figura 9: Evolução da Energia Armazenada - NE

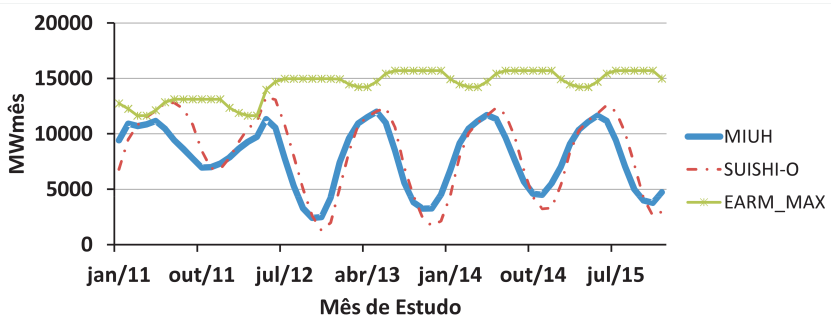

Figura 10: Evolução da Energia Armazenada - N

Para avaliar a variação da produtibilidade ao longo do período de estudo, as Figuras 18 e 19 mostram a evolução da altura de queda calculada pelos modelos MIUH e SUISHI-O. É possível verificar que o modelo proposto apresentou uma maior altura de queda líquida resultando numa maior produtividade para ambas as usinas com reservatório.

A mostra a evolução do volume armazenado final das usinas obtidas pelas metodologias. O MIUH manteve o armazenamento maior nas usinas a fim de obter maior produtibilidade das unidades de geração.

O modelo MIUH foi executado em computador com processador Quad core 2.66Ghz, 4GB de memória RAM e sistema

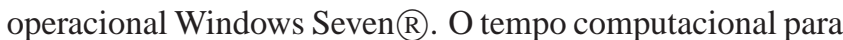
a simulação de cada cenário de afluência nos cinco anos de estudo foi de aproximadamente 120 segundos. O modelo SUISHI-O executado nas mesmas condições requer 15 segundos. Contudo, deve ser ressaltado que as metodologias são diferentes, o MIUH é baseado em algortimos de otimização não linear que requerem esforço computacional superior às heurísticas utilizadas pelo modelo SUISHI-O, que não garantem as condições de otimalidade.

\section{CONCLUSÃO}

Este trabalho apresentou um novo modelo para o planejamento individualizado da operação de médio/longo prazo de sistemas hidrotérmicos.

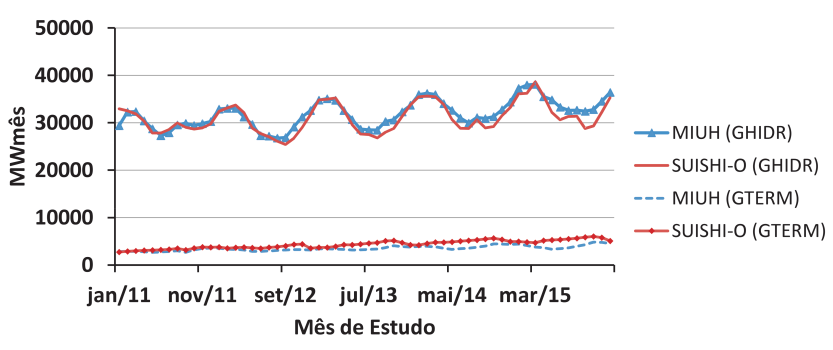

Figura 11: Geração Térmica e Hidráulica - SE

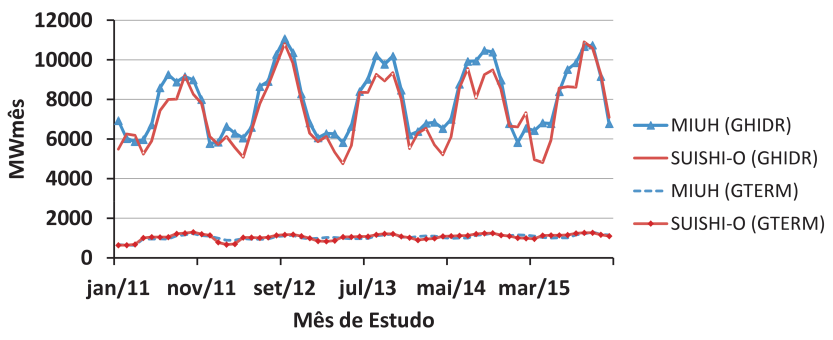

Figura 12: Geração Térmica e Hidráulica - S

O problema foi desacoplado em estágios mensais através da utilização de funções de custo futuro obtidas através de um modelo de decisão estratégica. Nos resultados apresentados, estas funções foram geradas pelo modelo NEWAVE, outros modelos de decisão estratégicas poderiam ser utilizados.

Para tanto, foi utilizado uma modelagem não linear do problema que leva em consideração todas as restrições físicas e operacionais do sistema, entre elas podem ser destacadas:

- Representação da não-linearidade da produtibilidade das usinas através da representação dos polinômios de cota jusante - vazão defluente $(P C J)$ e cota montante volume armazenado $(P C M)$;

- Utilização da função sigmoide na representação do $P C J$, o que permitiu uma melhor performance do modelo de otimização;

- Representação da restrição de volume mínimo para o vertimento para considerar-se a altura da cota do vertedouro;

- Consideração da defluência mínima das UHEs;

- Acoplamento em um único problema de otimização dos despacho mensal às usinas individualizadas com funções de custo futuro baseadas em subsistemas equivalentes;

- Consideração do volume evaporado e perdas hidráulicas; 


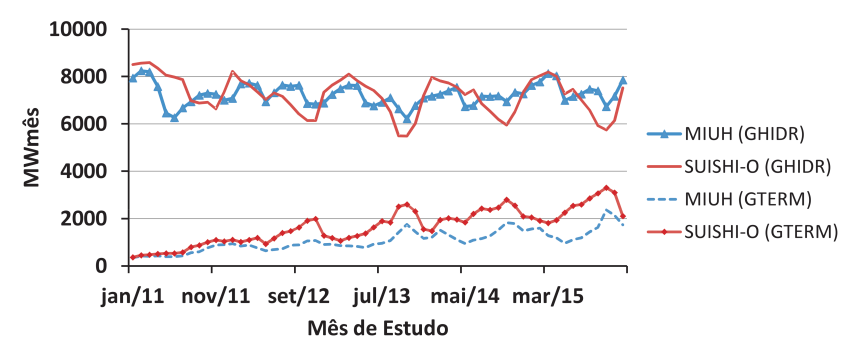

Figura 13: Geração Térmica e Hidráulica - NE

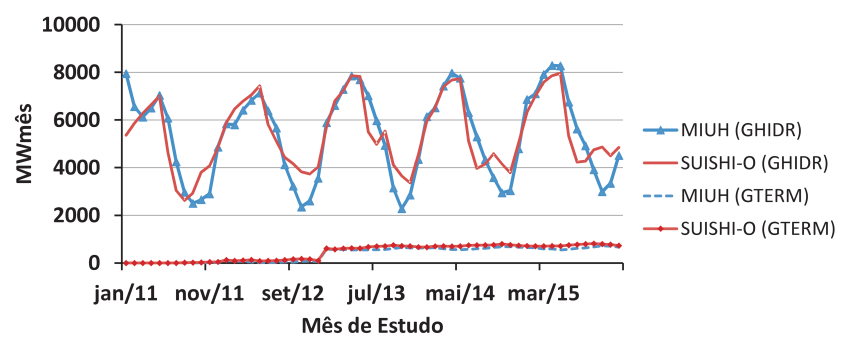

Figura 14: Evolução da Geração Térmica e Hidráulica - N

- Consideração dos limites de intercâmbio entre subsistemas equivalentes

Por outro lado, é importante destacar que a modelagem do problema baseada em otimização não linear, proposta neste trabalho, mostrou-se mais eficiente que a modelagem baseada em heurísticas. Neste contexto, verificou-se que o comportamento do custo operacional no horizonte de estudo foi inferior e menos volátil em relação ao modelo SUISHI-O.

No entanto, em linhas gerais, é importante ressaltar a robustez e coerência dos resultados do modelo proposto em relação ao SUISHI-O.

A partir dos resultados, verifica-se que o modelo proposto tem grande potencial para se tornar uma valiosa ferramenta de apoio no ambiente de operação e planejamento do Sistema Elétrico Brasileiro.

\section{AGRADECIMENTOS}

Os autores gostariam de agradecer ao CNPq, à CAPES, FAPEMIG e à ANEEL, juntamente com as empresas que financiam o P\&D Estratégico no tema "Modelos de Despacho Hidrotérmico", pelo apoio no desenvolvimento do presente trabalho.

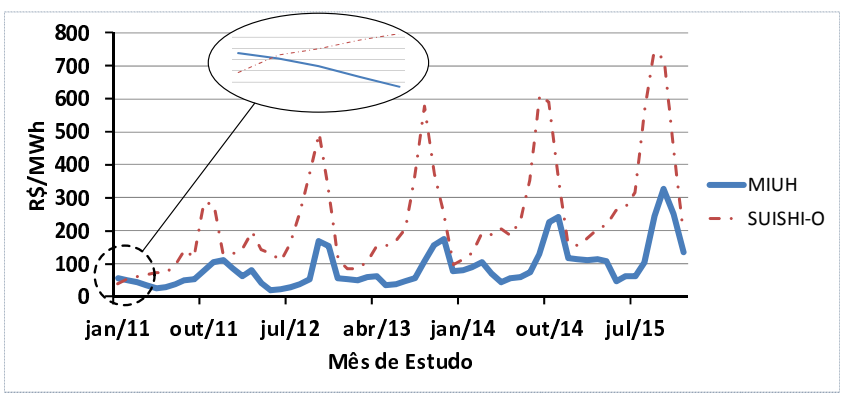

Figura 15: Evolução do Custo Marginal de Operação - SE

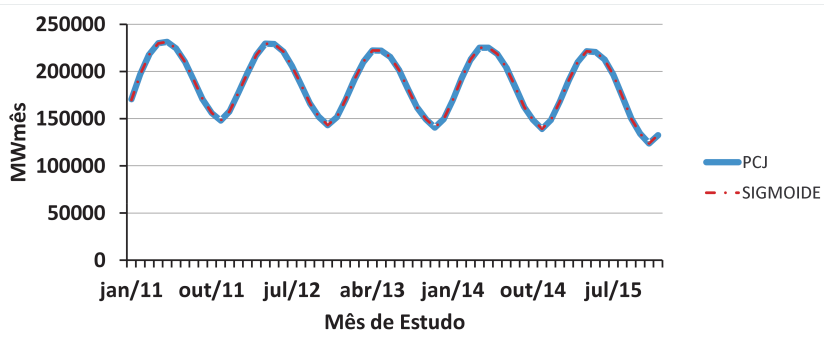

Figura 16: Comparação entre Sigmoide e Polinômio

\section{REFERÊNCIAS BIBLIOGRÁFICAS}

Arvanitidis, N. V e Rosing, J., (1970), "Composite Representation of a Multireservoir Hydroelectric Power System", IEEE Transactions on Power Apparatus and Systems PAS-89, pages 319-326.

Azevedo, A. T., Oliveira, A.R.L. e Soares, S. (2009). "Interior point method for long-term generation scheduling of large-scale hydrothermal systems"; Annals of operations research, 169, 55-80.

Bellman, R., (1957). Dynamic Programming, Princeton Princeton University Press.

J.F. Benders, "Partitioning Procedure for Solving Mixed Variables Programming Problems", Numerische Mathematik, Vol. 4, pp. 238-262, 1962.

Birge J.R. e Louveaux F. Introduction to stochastic programming. 1st ed. New York: Springer; 1997.

CEPEL, (2001), "Manual de Referência - Modelo NEWAVE", Relatório Técnico, CEPEL, Rio de Janeiro, RJ.

CEPEL, (2007), "Manual de Referência do Programa SUISHI-O 6.10 - Modelo de Simulação à Usinas Individualizadas para Subsistemas Hidrotérmicos Interligados", Relatório Técnico DP/DEA 51566/07 e 51572/07, CEPEL, Rio de Janeiro, RJ. 


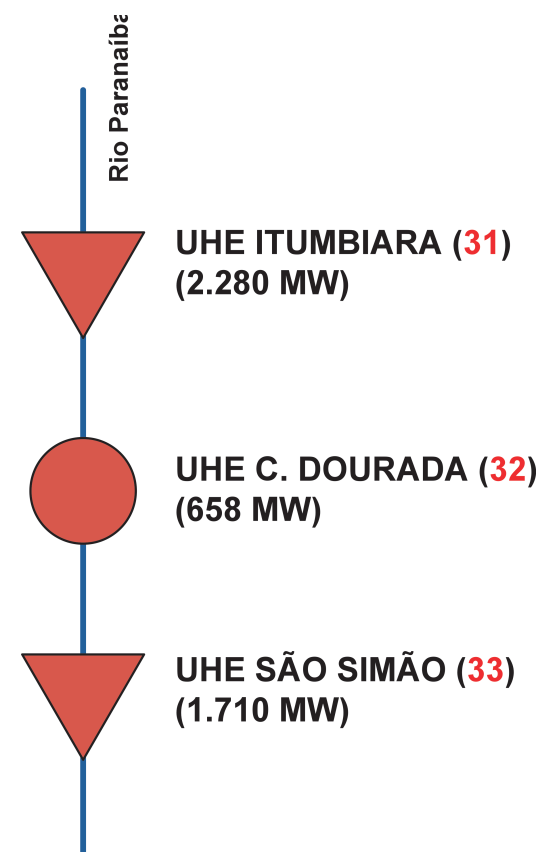

Figura 17: Topologia das Usinas

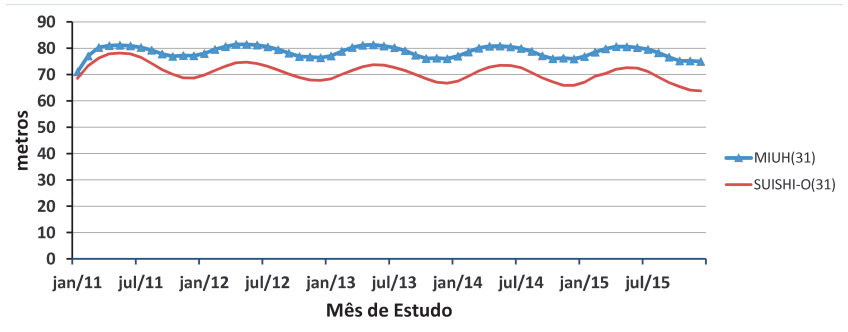

Figura 18: Altura de Queda - UHE ITUMBIARA

Dias, B. H.; Marcato, A. L. M.; Souza, R. C., Soares, M. P.; Silva Jr, I. C.; Oliveira, E. J.; Brandi, R. B. S.; e Ramos, T. P.; (2010), "Stochastic Dynamic Programming Applied to Hydrothermal Power Systems Operation Planning Based on the Convex Hull Algorithm", Mathematical Problems in Engineering, vol. 2010.

Fortunato, L.A.M., Neto, A. e Alencar, T. (1990). Introdução ao Planejamento da Expansão e Operação de Sistemas de Produção de Energia Elétrica, EDUFF, Niterói $-\mathrm{RJ}$.

Haykin, S., (2004); Neural Networks - A Comprehensive Foundation. McMillan, New York.

HIDALGO, I. G. , (2009); Fontane, Darrell G. ; Soares F., Secundino ; Cicogna, Marcelo A. ; Lopes, J. E. G. . Data Consolidation from Hydroelectric Plants. Journal of Energy Engineering

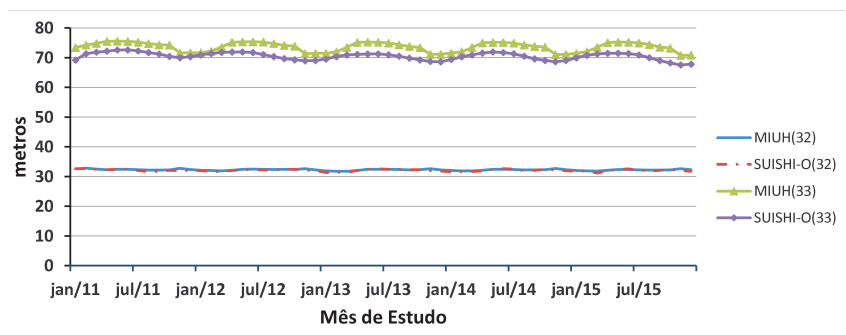

Figura 19: Altura de Queda

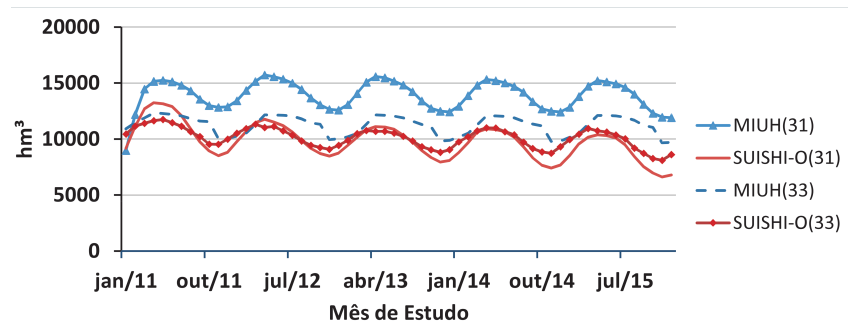

Figura 20: Evolução do Volume Armazenado

LINGO, (2008), “LINGO - User's Guide”, LINDO Systems Inc, Chicago, Illinois.

Marcato, A. L. M., (2002), Representação Híbrida de Sistemas Equivalentes e Individualizados para o Planejamento da Operação de Médio Prazo de Sistemas de Potência de Grande Porte, Tese de Doutorado, PUC-Rio, Rio de Janeiro.

ONS, (2011), Site oficial do Operador Nacional do Sistema, $\langle h t t p$ : //www.ons.org.br $>$, acessado em janeiro de 2011.

Pereira, M. V. F., (1989) "Optimal Stochastic Operation of Large Hidro-Electric Systems", Electrical Power and Energy Systems, vol.11.

Saad, M., Turgeon, A.; Bigras, P. e Duquette, R. (1994), Learning disaggregation technique for the operation of long-term hydroelectric power systems, Water Resources Research, 30(11), 3195-3202.

Santos, M. L. L., Silva, E. L., Finardi, E. C. e Gonçalves, R. E. C. (2009); "Practical aspects in solving the medium-term operation planning problem of hydrothermal power systems by using the progressive hedging method", Electrical Power and Energy Systems, 31, $546-552$.

Secundino, S. e Carneiro A. A. F. M. (1993), "Reservoir Operation Rules for Hidroeletric Power System Optimization", Proceedings of the PowerTech, Vol II, pp. 965-969, Athens. 
Silva, E. L. (2001). Formação de Preços em Mercados de Energia Elétrica. Editora Sagra Luzzatto, Porto Alegre - RS.

Silva, E. L. e Finardi, E. C. (2003); "Parallel Processing Applied to the Planning of Hydrothermal Systems". IEEE Transactions Parallel and Distributed Systems;14(8):721-9.

Turgeon, A., e Charbonneau, R. (1998). An AggregationDisaggregation Approach to Long-Term Reservoir Management. Water Resources Research, 34(12), 35853594.

Valdés, J. B., Filippo, J. M. D., Strzepek, K. M., e Restrepo, P. J. (1992). Aggregation-disaggregation approach to multireservoir operation. Journal of Water Resource Planning and Management, 118 (4), 423-444.

Zambelli, M., Toscano, A., Soares Filho, S., Santos E. e Nogueira, L. (2010), "Newave versus ODIN comparação entre modelo estocástico e determinístico no planejamento da operação energética do sistema interligado nacional", Congresso Brasileiro de Automática, CBA2010, pp. 347-354, Bonito, Brasil.

\section{APÊNDICE A}

A Tabela A1 apresenta os parâmetros da função sigmoide utilizada para representar os polinômios vazão defluente-nível jusante. A primeira coluna da tabela representa o código das usinas hidrelétricas e as demais colunas os coeficientes da Equação 15.

\begin{tabular}{|c|c|c|c|c|}
\hline$\Gamma a b$ & A1 & \multicolumn{2}{|c|}{ Parâmetros } & Sigmoides \\
\hline N. & $\mathrm{C}$ & A & B & M \\
\hline 1 & $8.861000 \mathrm{E}+02$ & $8.861000 \mathrm{E}+02$ & $-2.628106 \mathrm{E}-05$ & $-1.114694 \mathrm{E}+00$ \\
\hline 2 & $8.486091 \mathrm{E}+02$ & $8.605492 \mathrm{E}+02$ & $-2.530410 \mathrm{E}-03$ & $-2.087160 \mathrm{E}+02$ \\
\hline 4 & $7.680000 \mathrm{E}+02$ & $7.680000 \mathrm{E}+02$ & $-4.193110 \mathrm{E}-07$ & $-3.938796 \mathrm{E}-01$ \\
\hline 6 & $6.715328 \mathrm{E}+02$ & $6.723563 \mathrm{E}+02$ & $-4.500813 \mathrm{E}-03$ & $3.248942 \mathrm{E}+02$ \\
\hline 7 & $6.113593 \mathrm{E}+02$ & $6.306017 \mathrm{E}+02$ & $-3.346152 \mathrm{E}-04$ & $1.029742 \mathrm{E}+03$ \\
\hline 8 & $5.488359 \mathrm{E}+02$ & $5.632120 \mathrm{E}+02$ & $-3.179504 \mathrm{E}-04$ & $-6.414957 \mathrm{E}+02$ \\
\hline 9 & $5.030764 \mathrm{E}+02$ & $5.233948 \mathrm{E}+02$ & $-3.237031 \mathrm{E}-04$ & $1.934510 \mathrm{E}+03$ \\
\hline 10 & $4.932239 \mathrm{E}+02$ & $4.957665 \mathrm{E}+02$ & $-1.019704 \mathrm{E}-03$ & $3.941968 \mathrm{E}+02$ \\
\hline 11 & $4.594422 \mathrm{E}+02$ & 4.709374E+02 & $-2.755128 \mathrm{E}-04$ & $-1.097397 \mathrm{E}+03$ \\
\hline 12 & $4.454070 \mathrm{E}+02$ & $4.476096 \mathrm{E}+02$ & $-3.544235 \mathrm{E}-04$ & $2.308527 \mathrm{E}+03$ \\
\hline 14 & $7.436130 \mathrm{E}+02$ & $7.557737 \mathrm{E}+02$ & $-2.379605 \mathrm{E}-02$ & $2.608533 \mathrm{E}+01$ \\
\hline 15 & $-8.352786 \mathrm{E}+11$ & $5.812946 \mathrm{E}+02$ & $-6.710083 \mathrm{E}-03$ & $-3.766435 E+03$ \\
\hline 16 & $-2.019475 \mathrm{E}+10$ & $5.498161 \mathrm{E}+02$ & $-3.423489 \mathrm{E}-03$ & $-6.497850 \mathrm{E}+03$ \\
\hline 17 & 3.781631E+02 & $3.881568 \mathrm{E}+02$ & $-5.511797 \mathrm{E}-04$ & $1.507389 \mathrm{E}+03$ \\
\hline 18 & $3.279769 \mathrm{E}+02$ & 3.229808E+02 & $-1.293710 \mathrm{E}-02$ & $-1.162081 E+00$ \\
\hline
\end{tabular}

\begin{tabular}{|c|c|c|c|c|}
\hline N. & $\mathrm{C}$ & A & B & $\mathrm{M}$ \\
\hline 20 & $6.613282 \mathrm{E}+02$ & $7.617091 \mathrm{E}+02$ & $-1.872974 \mathrm{E}-03$ & $-1.328857 \mathrm{E}+03$ \\
\hline 21 & $6.746280 \mathrm{E}+02$ & $6.966217 \mathrm{E}+02$ & $-6.652465 \mathrm{E}-03$ & $8.736729 \mathrm{E}+02$ \\
\hline 24 & $5.085010 \mathrm{E}+02$ & $5.325683 \mathrm{E}+02$ & $-5.910859 \mathrm{E}-04$ & $3.172664 \mathrm{E}+02$ \\
\hline 25 & $-7.740181 \mathrm{E}+06$ & $7.051082 \mathrm{E}+02$ & $-7.303117 \mathrm{E}-04$ & $-1.839701 \mathrm{E}+04$ \\
\hline 26 & $6.128458 \mathrm{E}+02$ & $6.273941 \mathrm{E}+02$ & $-7.232640 \mathrm{E}-04$ & $-1.950858 \mathrm{E}+03$ \\
\hline 27 & $5.356208 \mathrm{E}+02$ & $5.719047 \mathrm{E}+02$ & $-9.294420 \mathrm{E}-04$ & $-1.493195 \mathrm{E}+03$ \\
\hline 28 & $-1.687233 \mathrm{E}+10$ & $5.235889 \mathrm{E}+02$ & $-6.080963 \mathrm{E}-04$ & $-3.570138 \mathrm{E}+04$ \\
\hline 29 & $7.414017 \mathrm{E}+02$ & $7.783675 \mathrm{E}+02$ & $-2.095123 \mathrm{E}-03$ & $-5.443298 \mathrm{E}+02$ \\
\hline 30 & $4.944544 \mathrm{E}+02$ & $5.192073 \mathrm{E}+02$ & $-2.810659 \mathrm{E}-03$ & $-2.523474 \mathrm{E}+02$ \\
\hline 31 & $4.328068 \mathrm{E}+02$ & $4.345933 \mathrm{E}+02$ & $-3.502393 \mathrm{E}-03$ & $4.491873 \mathrm{E}+02$ \\
\hline 32 & $3.971508 \mathrm{E}+02$ & $4.009294 \mathrm{E}+02$ & $-2.145984 \mathrm{E}-03$ & $2.269160 \mathrm{E}+02$ \\
\hline 33 & $3.227689 \mathrm{E}+02$ & $3.240259 \mathrm{E}+02$ & $-1.877034 \mathrm{E}-03$ & $-8.040120 \mathrm{E}-01$ \\
\hline 37 & $4.278217 \mathrm{E}+02$ & $4.274833 \mathrm{E}+02$ & $-3.824975 \mathrm{E}-02$ & $-3.481558 \mathrm{E}+00$ \\
\hline 38 & $4.039870 \mathrm{E}+02$ & $4.040766 \mathrm{E}+02$ & $-3.826996 \mathrm{E}-02$ & $1.275355 \mathrm{E}+02$ \\
\hline 39 & $3.796072 \mathrm{E}+02$ & $3.797076 \mathrm{E}+02$ & $-9.391535 \mathrm{E}-02$ & $1.358053 \mathrm{E}+02$ \\
\hline 40 & $3.579742 \mathrm{E}+02$ & $3.580116 \mathrm{E}+02$ & $1.199685 \mathrm{E}-02$ & $-2.205960 \mathrm{E}+00$ \\
\hline 42 & $-4.981299 \mathrm{E}+11$ & $3.289273 \mathrm{E}+02$ & $-5.060919 \mathrm{E}-04$ & $-4.988798 \mathrm{E}+04$ \\
\hline 44 & $2.798890 \mathrm{E}+02$ & $2.805436 \mathrm{E}+02$ & $-9.229306 \mathrm{E}-04$ & $2.700389 \mathrm{E}+03$ \\
\hline 45 & $2.540274 \mathrm{E}+02$ & $2.595948 \mathrm{E}+02$ & $-4.611914 \mathrm{E}-04$ & $5.444436 \mathrm{E}+03$ \\
\hline 46 & $-4.241441 \mathrm{E}+10$ & $2.437381 \mathrm{E}+02$ & $-7.704484 \mathrm{E}-05$ & $-2.888086 \mathrm{E}+05$ \\
\hline 47 & $5.015213 \mathrm{E}+02$ & $5.372907 \mathrm{E}+02$ & $-7.713425 \mathrm{E}-04$ & $-2.115046 \mathrm{E}+03$ \\
\hline 48 & $-1.995593 \mathrm{E}+11$ & $5.089235 \mathrm{E}+02$ & $-9.950001 \mathrm{E}-04$ & $-2.454905 \mathrm{E}+04$ \\
\hline 49 & $3.769952 \mathrm{E}+02$ & $4.036399 \mathrm{E}+02$ & $-8.490305 \mathrm{E}-04$ & $-1.311642 \mathrm{E}+03$ \\
\hline 50 & $3.587638 \mathrm{E}+02$ & 3.742015E+02 & $-4.928777 \mathrm{E}-04$ & $4.916416 \mathrm{E}+02$ \\
\hline 51 & $3.459145 \mathrm{E}+02$ & $3.565132 \mathrm{E}+02$ & $-4.827484 \mathrm{E}-04$ & $1.803127 \mathrm{E}+02$ \\
\hline 52 & $-9.595769 \mathrm{E}+09$ & $3.369341 \mathrm{E}+02$ & $-5.528206 \mathrm{E}-04$ & $-3.928744 \mathrm{E}+04$ \\
\hline 61 & $2.790573 \mathrm{E}+02$ & $2.892291 \mathrm{E}+02$ & $-4.700583 \mathrm{E}-04$ & $5.370551 \mathrm{E}+02$ \\
\hline 62 & $-3.644284 \mathrm{E}+05$ & $2.650480 \mathrm{E}+02$ & $-1.599857 \mathrm{E}-04$ & $-6.738070 \mathrm{E}+04$ \\
\hline 63 & $2.104484 \mathrm{E}+02$ & $2.456200 \mathrm{E}+02$ & $-2.113257 \mathrm{E}-04$ & $-4.159282 \mathrm{E}+03$ \\
\hline 66 & $1.000000 \mathrm{E}+02$ & $1.000000 \mathrm{E}+02$ & $-1.331823 \mathrm{E}-07$ & $-1.033934 \mathrm{E}-03$ \\
\hline 117 & $7.199998 \mathrm{E}+02$ & $7.199999 \mathrm{E}+02$ & $-7.296820 \mathrm{E}-04$ & $-4.064309 \mathrm{E}+01$ \\
\hline 118 & $7.287499 \mathrm{E}+02$ & $7.287500 \mathrm{E}+02$ & $-1.243563 \mathrm{E}-04$ & $-2.081891 \mathrm{E}+01$ \\
\hline 119 & $1.150000 \mathrm{E}+01$ & $1.150000 \mathrm{E}+01$ & $-9.301251 \mathrm{E}-07$ & $-2.920658 \mathrm{E}-04$ \\
\hline 120 & $5.510121 \mathrm{E}+02$ & $5.617088 \mathrm{E}+02$ & $-1.810910 \mathrm{E}-02$ & $2.087694 \mathrm{E}+01$ \\
\hline 121 & $-3.270972 \mathrm{E}+05$ & $6.276336 \mathrm{E}+02$ & $-7.407334 \mathrm{E}-03$ & $-1.548546 \mathrm{E}+03$ \\
\hline 122 & $5.772000 \mathrm{E}+02$ & $5.772000 \mathrm{E}+02$ & $-1.087150 \mathrm{E}-04$ & $-5.912675 \mathrm{E}-01$ \\
\hline 123 & $3.919989 \mathrm{E}+02$ & $3.957555 \mathrm{E}+02$ & $-9.539563 \mathrm{E}-03$ & $1.231179 \mathrm{E}+02$ \\
\hline 124 & $9.030000 \mathrm{E}+01$ & $9.030000 \mathrm{E}+01$ & 9.488575E-04 & $\begin{array}{l}-3.196610 \mathrm{E}-01 \\
\end{array}$ \\
\hline 126 & $5.222689 \mathrm{E}+02$ & $5.300699 \mathrm{E}+02$ & $-9.239046 \mathrm{E}-03$ & $2.913039 \mathrm{E}+01$ \\
\hline 127 & $3.560000 \mathrm{E}+02$ & $3.560000 \mathrm{E}+02$ & $-3.331878 \mathrm{E}-06$ & $-2.829828 \mathrm{E}-01$ \\
\hline 129 & $1.009263 \mathrm{E}+02$ & 1.465050E+02 & $-5.223516 \mathrm{E}-04$ & $-2.829937 \mathrm{E}+03$ \\
\hline 130 & $8.933805 \mathrm{E}+01$ & $1.050472 \mathrm{E}+02$ & $-2.276420 \mathrm{E}-03$ & $-3.248204 \mathrm{E}+02$ \\
\hline 131 & $8.689999 \mathrm{E}+01$ & $8.689999 \mathrm{E}+01$ & $-1.930103 \mathrm{E}-06$ & $-5.745671 \mathrm{E}-03$ \\
\hline 132 & $9.030000 \mathrm{E}+01$ & $9.030000 \mathrm{E}+01$ & $-4.749940 \mathrm{E}-06$ & $-1.681885 \mathrm{E}-02$ \\
\hline 133 & 4.171983E+01 & $5.126668 \mathrm{E}+01$ & $-4.051968 \mathrm{E}-03$ & $-2.032827 \mathrm{E}+01$ \\
\hline 134 & $2.541604 \mathrm{E}+02$ & $2.577013 \mathrm{E}+02$ & $-2.190178 \mathrm{E}-02$ & $1.660834 \mathrm{E}+02$ \\
\hline 135 & $2.040000 \mathrm{E}+02$ & $2.040000 \mathrm{E}+02$ & $-2.265711 \mathrm{E}-06$ & $-4.097328 \mathrm{E}-02$ \\
\hline 139 & $2.391463 \mathrm{E}+02$ & $2.854229 \mathrm{E}+02$ & $-9.533474 \mathrm{E}-04$ & $-1.424073 \mathrm{E}+03$ \\
\hline 141 & $1.496879 \mathrm{E}+02$ & $1.721938 \mathrm{E}+02$ & $-3.983146 \mathrm{E}-04$ & $-1.913932 \mathrm{E}+03$ \\
\hline
\end{tabular}

606 Revista Controle \& Automação/Vol.23 no.5/Setembro e Outubro 2012 


\begin{tabular}{|c|c|c|c|c|}
\hline N. & $\mathrm{C}$ & A & B & M \\
\hline 143 & $-5.057291 \mathrm{E}+11$ & $6.668258 \mathrm{E}+01$ & $-3.085094 \mathrm{E}-04$ & $-8.090245 E+04$ \\
\hline 144 & $1.703576 \mathrm{E}+01$ & $5.095816 \mathrm{E}+01$ & $-3.242321 \mathrm{E}-04$ & $-1.091156 \mathrm{E}+03$ \\
\hline 148 & $-1.430559 \mathrm{E}+12$ & $3.377952 \mathrm{E}+02$ & $-2.397036 \mathrm{E}-03$ & $-1.069711 \mathrm{E}+04$ \\
\hline 153 & $3.009980 \mathrm{E}+02$ & $3.123436 \mathrm{E}+02$ & $-1.003081 \mathrm{E}-02$ & $-1.129011 \mathrm{E}+01$ \\
\hline 155 & $5.648070 \mathrm{E}+02$ & $5.851517 \mathrm{E}+02$ & $-1.323207 \mathrm{E}-03$ & $-3.095827 \mathrm{E}+02$ \\
\hline 156 & $4.929305 \mathrm{E}+02$ & $5.202967 \mathrm{E}+02$ & $-3.209572 \mathrm{E}-04$ & $-4.260774 \mathrm{E}+03$ \\
\hline 162 & $-1.984086 \mathrm{E}+06$ & $6.420545 \mathrm{E}+02$ & $-3.534002 \mathrm{E}-03$ & $-3.615411 \mathrm{E}+03$ \\
\hline 192 & $3.755000 \mathrm{E}+02$ & $3.755000 \mathrm{E}+02$ & $1.807500 \mathrm{E}-05$ & $-1.394506 \mathrm{E}-01$ \\
\hline 193 & $2.551000 \mathrm{E}+02$ & $2.551000 \mathrm{E}+02$ & $-2.904931 \mathrm{E}-05$ & $-6.436181 \mathrm{E}-02$ \\
\hline 195 & $2.482091 \mathrm{E}+02$ & $2.490978 \mathrm{E}+02$ & $-3.331690 \mathrm{E}-02$ & $1.003397 \mathrm{E}+02$ \\
\hline 196 & $2.916837 \mathrm{E}+02$ & $3.071841 \mathrm{E}+02$ & $-2.002164 \mathrm{E}-02$ & $-3.788356 \mathrm{E}+01$ \\
\hline 203 & $-7.630448 \mathrm{E}+06$ & $7.345561 \mathrm{E}+02$ & $-1.757546 \mathrm{E}-03$ & $-7.976809 \mathrm{E}+03$ \\
\hline 217 & $3.598254 \mathrm{E}+02$ & $4.638458 \mathrm{E}+02$ & $-3.521875 \mathrm{E}-02$ & $1.899952 \mathrm{E}+02$ \\
\hline 228 & $-1.300853 \mathrm{E}+11$ & $2.611640 \mathrm{E}+02$ & $-1.794374 \mathrm{E}-04$ & $-1.271601 \mathrm{E}+05$ \\
\hline 241 & $3.097310 \mathrm{E}+02$ & $3.304107 \mathrm{E}+02$ & $-2.987196 \mathrm{E}-03$ & $-5.504603 \mathrm{E}+02$ \\
\hline 249 & $3.492805 \mathrm{E}+02$ & $3.955528 \mathrm{E}+02$ & $-3.734675 \mathrm{E}-04$ & $-3.533932 \mathrm{E}+03$ \\
\hline 251 & $3.306039 \mathrm{E}+02$ & $3.394646 \mathrm{E}+02$ & $-6.917555 \mathrm{E}-04$ & $1.549693 \mathrm{E}+03$ \\
\hline 252 & $2.508135 \mathrm{E}+02$ & $2.947500 \mathrm{E}+02$ & $-5.283083 \mathrm{E}-04$ & $-2.169205 \mathrm{E}+03$ \\
\hline 253 & $2.624185 \mathrm{E}+02$ & $2.660205 \mathrm{E}+02$ & $-1.262689 \mathrm{E}-03$ & $2.168872 \mathrm{E}+03$ \\
\hline 257 & $2.318780 \mathrm{E}+02$ & $2.377040 \mathrm{E}+02$ & $-6.374051 \mathrm{E}-04$ & $1.249450 \mathrm{E}+03$ \\
\hline 261 & $-2.794640 \mathrm{E}+03$ & $1.929455 \mathrm{E}+02$ & $-1.114839 \mathrm{E}-04$ & $-4.417405 \mathrm{E}+04$ \\
\hline 262 & $3.899539 \mathrm{E}+02$ & $4.021651 \mathrm{E}+02$ & $-3.824512 \mathrm{E}-03$ & $-3.591035 \mathrm{E}+01$ \\
\hline 276 & $-3.127193 \mathrm{E}+03$ & $2.080904 \mathrm{E}+02$ & $-4.890613 \mathrm{E}-03$ & $-1.308065 E+03$ \\
\hline 278 & $2.227630 \mathrm{E}+02$ & $2.272475 \mathrm{E}+02$ & $-3.983819 \mathrm{E}-03$ & $2.484805 \mathrm{E}+02$ \\
\hline 279 & $-1.511933 \mathrm{E}+01$ & $6.543211 \mathrm{E}+01$ & $-1.090647 \mathrm{E}-03$ & $-1.442027 \mathrm{E}+03$ \\
\hline 281 & $1.456227 \mathrm{E}+02$ & $1.505121 \mathrm{E}+02$ & $-1.495654 \mathrm{E}-02$ & $3.005710 \mathrm{E}+01$ \\
\hline 283 & $-1.208715 \mathrm{E}+11$ & $3.939045 \mathrm{E}+01$ & $-1.010281 \mathrm{E}-03$ & $-2.327388 \mathrm{E}+04$ \\
\hline 285 & $6.965929 \mathrm{E}+01$ & $7.389574 \mathrm{E}+01$ & $-1.971009 \mathrm{E}-04$ & $1.380372 \mathrm{E}+04$ \\
\hline 287 & $-1.909423 \mathrm{E}+12$ & $6.430345 \mathrm{E}+01$ & $-3.465903 \mathrm{E}-05$ & $-7.280300 \mathrm{E}+05$ \\
\hline 290 & $5.142811 \mathrm{E}+02$ & $5.312885 \mathrm{E}+02$ & $-2.044032 \mathrm{E}-02$ & $-5.361571 \mathrm{E}+01$ \\
\hline 304 & $3.200000 \mathrm{E}+02$ & $3.200000 \mathrm{E}+02$ & $-4.766332 \mathrm{E}-06$ & $-3.497130 \mathrm{E}-01$ \\
\hline 305 & $-1.368850 \mathrm{E}+10$ & $1.823873 \mathrm{E}+02$ & $-9.003081 \mathrm{E}-03$ & $-2.463211 \mathrm{E}+03$ \\
\hline 310 & $1.097912 \mathrm{E}+02$ & $1.214651 \mathrm{E}+02$ & $-1.468211 \mathrm{E}-03$ & $6.948539 \mathrm{E}+02$ \\
\hline 311 & $4.461327 \mathrm{E}+02$ & $4.514772 \mathrm{E}+02$ & $-3.754310 \mathrm{E}-03$ & $1.808378 \mathrm{E}+02$ \\
\hline 312 & $4.112537 \mathrm{E}+02$ & $4.128860 \mathrm{E}+02$ & $-3.394852 \mathrm{E}-03$ & $4.970380 \mathrm{E}+02$ \\
\hline 315 & $-2.719632 \mathrm{E}+10$ & $3.298651 \mathrm{E}+02$ & $-1.658741 \mathrm{E}-03$ & $-1.343236 \mathrm{E}+04$ \\
\hline 319 & $5.100336 \mathrm{E}+02$ & $5.108301 \mathrm{E}+02$ & $-4.366617 \mathrm{E}-02$ & $1.356860 \mathrm{E}+01$ \\
\hline 57 & $-6.796397 \mathrm{E}+11$ & $5.201265 \mathrm{E}+02$ & $-8.065349 \mathrm{E}-04$ & $-3.096339 \mathrm{E}+04$ \\
\hline 71 & $6.971308 \mathrm{E}+02$ & $7.097020 \mathrm{E}+02$ & $-1.889360 \mathrm{E}-03$ & $-3.506046 \mathrm{E}+02$ \\
\hline 72 & $5.981542 \mathrm{E}+02$ & $6.111695 \mathrm{E}+02$ & $-7.205347 \mathrm{E}-03$ & $-1.608553 \mathrm{E}+01$ \\
\hline 73 & $6.020000 \mathrm{E}+02$ & $6.020000 \mathrm{E}+02$ & $-6.363224 \mathrm{E}-05$ & $-8.524291 \mathrm{E}+00$ \\
\hline 74 & $6.010430 \mathrm{E}+02$ & $6.059560 \mathrm{E}+02$ & $-1.418448 \mathrm{E}-03$ & $1.090360 \mathrm{E}+03$ \\
\hline 76 & $4.891930 \mathrm{E}+02$ & $4.996804 \mathrm{E}+02$ & $-5.693194 \mathrm{E}-04$ & $4.572581 \mathrm{E}+03$ \\
\hline 77 & $3.636604 \mathrm{E}+02$ & $4.161687 \mathrm{E}+02$ & $-1.543201 \mathrm{E}-04$ & $-2.290651 \mathrm{E}+03$ \\
\hline 78 & $2.851987 \mathrm{E}+02$ & $3.361663 \mathrm{E}+02$ & $-2.153799 \mathrm{E}-04$ & $-4.372005 E+03$ \\
\hline 82 & $2.504375 \mathrm{E}+02$ & $2.646966 \mathrm{E}+02$ & $-1.623993 \mathrm{E}-04$ & $-6.614586 \mathrm{E}+02$ \\
\hline 83 & $2.356716 \mathrm{E}+02$ & $2.481592 \mathrm{E}+02$ & $-1.190896 \mathrm{E}-04$ & $2.845526 \mathrm{E}+03$ \\
\hline
\end{tabular}

\begin{tabular}{|c|c|c|c|c|}
\hline N. & $\mathrm{C}$ & A & B & M \\
\hline 86 & $4.771539 \mathrm{E}+02$ & $4.849625 \mathrm{E}+02$ & $-8.124174 \mathrm{E}-04$ & $7.007896 \mathrm{E}+02$ \\
\hline 89 & $6.566768 \mathrm{E}+02$ & $6.656508 \mathrm{E}+02$ & $-5.383089 \mathrm{E}-04$ & $1.005643 \mathrm{E}+03$ \\
\hline 90 & $-6.825630 \mathrm{E}+11$ & $4.816902 \mathrm{E}+02$ & $-6.716383 \mathrm{E}-04$ & $-3.698681 E+04$ \\
\hline 91 & $3.541805 \mathrm{E}+02$ & $3.865038 \mathrm{E}+02$ & $-2.252887 \mathrm{E}-04$ & $-9.113154 \mathrm{E}+02$ \\
\hline 92 & $-1.848040 \mathrm{E}+12$ & $2.763890 \mathrm{E}+02$ & $-1.365773 \mathrm{E}-04$ & $-1.872667 \mathrm{E}+05$ \\
\hline 93 & $3.304169 \mathrm{E}+02$ & $3.384745 \mathrm{E}+02$ & $-3.997571 \mathrm{E}-03$ & $-8.296478 \mathrm{E}+01$ \\
\hline 94 & $2.647965 \mathrm{E}+02$ & $2.677539 \mathrm{E}+02$ & $-8.999460 \mathrm{E}-03$ & $2.943369 \mathrm{E}+02$ \\
\hline 95 & $-1.267167 \mathrm{E}+10$ & $4.275492 \mathrm{E}+02$ & $-4.312016 \mathrm{E}-03$ & $-5.307529 \mathrm{E}+03$ \\
\hline 97 & $-6.520056 \mathrm{E}+11$ & $1.437558 \mathrm{E}+02$ & $-2.489434 \mathrm{E}-03$ & $-1.014680 \mathrm{E}+04$ \\
\hline 98 & $9.593424 \mathrm{E}+01$ & $1.155201 \mathrm{E}+02$ & $-6.959109 \mathrm{E}-04$ & $1.653834 \mathrm{E}+02$ \\
\hline 99 & $3.475803 \mathrm{E}+01$ & $7.722247 \mathrm{E}+01$ & $-6.459441 \mathrm{E}-04$ & $-1.872146 \mathrm{E}+03$ \\
\hline 101 & $1.268453 \mathrm{E}+02$ & $1.356123 \mathrm{E}+02$ & $-9.713427 \mathrm{E}-04$ & $2.561604 \mathrm{E}+02$ \\
\hline 102 & $8.830488 \mathrm{E}+01$ & $1.030067 \mathrm{E}+02$ & $-8.947576 \mathrm{E}-04$ & $-1.880292 \mathrm{E}+02$ \\
\hline 103 & $-4.220280 \mathrm{E}+11$ & $2.225680 \mathrm{E}+02$ & $-1.097658 \mathrm{E}-04$ & $-2.214640 \mathrm{E}+05$ \\
\hline 110 & 4.573499E+02 & $4.573499 \mathrm{E}+02$ & $-1.335140 \mathrm{E}-02$ & $-4.919815 \mathrm{E}+01$ \\
\hline 111 & $2.605440 \mathrm{E}+02$ & $2.854984 \mathrm{E}+02$ & $-9.963810 \mathrm{E}-04$ & $-8.142898 \mathrm{E}+02$ \\
\hline 112 & $1.640740 \mathrm{E}+02$ & $1.893568 \mathrm{E}+02$ & $-9.722069 \mathrm{E}-04$ & $-7.994121 \mathrm{E}+02$ \\
\hline 113 & $6.870589 \mathrm{E}+01$ & $1.001438 \mathrm{E}+02$ & $-8.714585 \mathrm{E}-04$ & $-1.194017 \mathrm{E}+03$ \\
\hline 114 & $-4.936888 \mathrm{E}+10$ & $6.064631 \mathrm{E}+01$ & $-5.584283 \mathrm{E}-04$ & $-4.056129 \mathrm{E}+04$ \\
\hline 115 & $9.070000 \mathrm{E}+01$ & $9.070000 \mathrm{E}+01$ & $-3.905086 \mathrm{E}-06$ & $-4.534225 \mathrm{E}-02$ \\
\hline 215 & $-8.105418 \mathrm{E}+10$ & $1.181995 \mathrm{E}+02$ & $-8.742688 \mathrm{E}-04$ & $-2.664397 \mathrm{E}+04$ \\
\hline 154 & $-7.010276 \mathrm{E}+11$ & $3.541270 \mathrm{E}+01$ & $-3.485297 \mathrm{E}-04$ & $-7.235094 \mathrm{E}+04$ \\
\hline 169 & $-4.530855 \mathrm{E}+11$ & $3.718514 \mathrm{E}+02$ & $-8.080143 \mathrm{E}-05$ & $-3.026851 \mathrm{E}+05$ \\
\hline 172 & $2.515000 \mathrm{E}+02$ & $2.515000 \mathrm{E}+02$ & $-5.306592 \mathrm{E}-08$ & $-1.435907 \mathrm{E}-03$ \\
\hline 176 & $1.380000 \mathrm{E}+02$ & $1.380000 \mathrm{E}+02$ & $-4.365070 \mathrm{E}-08$ & $-3.618048 \mathrm{E}-04$ \\
\hline 178 & $-1.118670 \mathrm{E}+11$ & $2.165263 \mathrm{E}+01$ & $-2.507375 \mathrm{E}-04$ & $-9.301275 \mathrm{E}+04$ \\
\hline 189 & $-3.041669 \mathrm{E}+06$ & $8.550248 \mathrm{E}+00$ & $-9.540037 \mathrm{E}-04$ & $-1.413066 \mathrm{E}+04$ \\
\hline 190 & $2.554991 \mathrm{E}+02$ & $2.665227 \mathrm{E}+02$ & $-1.097611 \mathrm{E}-03$ & $9.063222 \mathrm{E}+02$ \\
\hline 294 & $6.358765 \mathrm{E}+02$ & $6.367252 \mathrm{E}+02$ & $-1.421258 \mathrm{E}-01$ & $3.353665 \mathrm{E}+00$ \\
\hline 295 & $5.136329 \mathrm{E}+02$ & $5.161081 \mathrm{E}+02$ & $-2.492837 \mathrm{E}-03$ & $1.378222 \mathrm{E}+02$ \\
\hline 298 & $3.269666 \mathrm{E}+02$ & $3.276100 \mathrm{E}+02$ & $-1.645882 \mathrm{E}-01$ & $3.744958 \mathrm{E}+00$ \\
\hline 308 & $5.767937 \mathrm{E}+02$ & $5.773862 \mathrm{E}+02$ & $-4.704174 \mathrm{E}-02$ & $1.313814 \mathrm{E}+01$ \\
\hline 267 & $-4.402977 \mathrm{E}+12$ & $1.454380 \mathrm{E}+02$ & $-1.008246 \mathrm{E}-04$ & $-2.600200 \mathrm{E}+05$ \\
\hline 272 & $3.676378 \mathrm{E}+01$ & $5.181183 \mathrm{E}+01$ & $-3.581294 \mathrm{E}-03$ & $-3.167355 E+01$ \\
\hline 275 & $-3.757451 \mathrm{E}+12$ & $2.088823 \mathrm{E}+01$ & $-2.969739 \mathrm{E}-05$ & $-8.789914 \mathrm{E}+05$ \\
\hline 277 & $-1.993411 \mathrm{E}+01$ & $3.093660 \mathrm{E}+01$ & $-7.983303 \mathrm{E}-04$ & $-2.072714 \mathrm{E}+03$ \\
\hline 280 & $2.123294 \mathrm{E}+01$ & $2.226727 \mathrm{E}+01$ & $-8.782805 \mathrm{E}-03$ & $3.342600 \mathrm{E}+02$ \\
\hline 284 & $-1.266649 \mathrm{E}+02$ & $9.749299 \mathrm{E}+00$ & $-2.343241 \mathrm{E}-04$ & $-1.181895 \mathrm{E}+04$ \\
\hline 291 & $3.323915 \mathrm{E}+02$ & $3.336378 \mathrm{E}+02$ & $-4.492365 \mathrm{E}-03$ & $1.594590 \mathrm{E}+02$ \\
\hline 292 & $2.833679 \mathrm{E}+02$ & $2.854061 \mathrm{E}+02$ & $-8.655938 \mathrm{E}-03$ & $6.349545 \mathrm{E}+01$ \\
\hline 302 & $1.706970 \mathrm{E}+02$ & $1.725908 \mathrm{E}+02$ & $-5.141287 \mathrm{E}-03$ & $1.159636 \mathrm{E}+02$ \\
\hline 303 & $2.332608 \mathrm{E}+02$ & $2.343219 \mathrm{E}+02$ & $-3.662096 \mathrm{E}-03$ & $1.698923 \mathrm{E}+02$ \\
\hline 306 & $2.626394 \mathrm{E}+02$ & $2.627203 \mathrm{E}+02$ & $-4.100486 \mathrm{E}-03$ & $-1.947059 \mathrm{E}-01$ \\
\hline 314 & $-5.240520 \mathrm{E}+11$ & $8.980113 \mathrm{E}+01$ & $-7.634989 \mathrm{E}-05$ & $-3.267205 \mathrm{E}+05$ \\
\hline
\end{tabular}

